Erratum à L'algèbre de Hopf et le groupe de Galois motiviques, II

Ayoub, Joseph

Abstract: This is an erratum to http://dx.doi.org/10.1515/crelle-2012-0090.

DOI: https://doi.org/10.1515/crelle-2013-0018

Posted at the Zurich Open Repository and Archive, University of Zurich ZORA URL: https://doi.org/10.5167/uzh-155419

Journal Article

Published Version

Originally published at:

Ayoub, Joseph (2014). Erratum à L'algèbre de Hopf et le groupe de Galois motiviques, II. Journal für die Reine und Angewandte Mathematik, 2014(693):227-230.

DOI: https://doi.org/10.1515/crelle-2013-0018 


\section{Erratum à \\ L'algèbre de Hopf et le groupe de Galois motiviques d'un corps de caractéristique nulle, II}

(J. reine angew. Math. 693 (2014), 151-226)

Par Joseph Ayoub à Zürich

Il y a une erreur dans l'étape A de la preuve du théorème 2.49 de [1]. Il y est affirmé à tort que le monomorphisme de $\Lambda$-espaces vectoriels $\mathbb{Z}$-gradués

$$
\Gamma\left(C^{\text {an }}, \mathrm{H}_{\bullet}\left(\mathrm{Bti}_{C}^{*}\left(i^{*} M\right)\right)\right) \hookrightarrow \mathrm{H}_{\bullet}\left(\mathrm{R} f_{*}^{\text {an }} \mathrm{Bti}_{C}^{*}\left(i^{*} M\right)\right)
$$

était canonique, i.e., indépendant du choix d'un isomorphisme

$$
\mathrm{Bti}_{C}^{*}\left(i^{*} M\right) \simeq \bigoplus_{n \in \mathbb{Z}} \mathrm{H}_{n}\left(\mathrm{Bti}_{C}^{*}\left(i^{*} M\right)\right)[n]
$$

induisant l'identité en homologie. Ceci pose un problème lorsqu'on veut passer à la colimite suivant $i \in \mathcal{d}$ pour obtenir un monomorphisme

$$
\underset{i \in \mathcal{l}}{\operatorname{colim}} \Gamma\left(C^{\text {an }}, \mathrm{H}_{\bullet}\left(\mathrm{Bti}_{C}^{*}\left(i^{*} M\right)\right)\right) \hookrightarrow \underset{i \in \mathcal{l}}{\operatorname{colim}} \mathrm{H}_{\bullet}\left(\mathrm{R} f_{*}^{\text {an }} \mathrm{Bti}_{C}^{*}\left(i^{*} M\right)\right) .
$$

Voici comment on peut réparer ce problème. L'isomorphisme (1) induit un isomorphisme de $\Lambda$-espaces vectoriels $\mathbb{Z}$-gradués :

(2) $\mathrm{H}_{\bullet}\left(\mathrm{R} f_{*}^{\mathrm{an}} \mathrm{Bti}_{C}^{*}\left(i^{*} M\right)\right) \simeq \Gamma\left(C^{\mathrm{an}}, \mathrm{H}_{\bullet}\left(\mathrm{Bti}_{C}^{*}\left(i^{*} M\right)\right)\right) \oplus \mathrm{R}^{1} \Gamma\left(C^{\mathrm{an}}, \mathrm{H}_{\bullet+1}\left(\mathrm{Bti}_{C}^{*}\left(i^{*} M\right)\right)\right)$.

L'isomorphisme (1) étant bien défini modulo des flèches du type $\mathrm{H}_{n}(-)[n] \rightarrow \mathrm{H}_{n+1}(-)[n+1]$, l'épimorphisme

$$
\mathrm{H}_{\bullet}\left(\mathrm{R} f_{*}^{\mathrm{an}} \mathrm{Bti}_{C}^{*}\left(i^{*} M\right)\right) \rightarrow \Gamma\left(C^{\mathrm{an}}, \mathrm{H}_{\bullet}\left(\mathrm{Bti}_{C}^{*}\left(i^{*} M\right)\right)\right),
$$

déduit de la décomposition (2), est canonique. Plus précisément, il est fonctoriel en $\mathrm{Bti}_{C}^{*}\left(i^{*} M\right)$. On peut donc prendre sa colimite suivant $i \in \mathcal{d}$ pour obtenir un morphisme surjectif de $\Lambda$-espaces vectoriels $\mathbb{Z}$-gradués

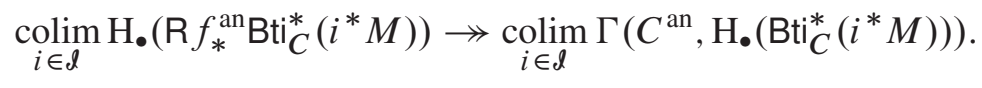

L'auteur a bénéficié du soutien partiel du Fond National Suisse de la Recherche Scientifique (NSF), projet no. 2000201-124737/1. 
Il est donc bien suffisant de montrer que le morphisme canonique $\Lambda \rightarrow p_{\sharp} \mathrm{R} f_{*}^{\text {an }} \mathrm{Bti}_{C}^{*}(M)$ est inversible et le reste de la preuve reste inchangé.

\section{Références}

[1] J. Ayoub, L'algèbre de Hopf et le groupe de Galois motiviques d'un corps de caractéristique nulle, II, J. reine angew. Math. 693 (2014), 151-226.

Joseph Ayoub, Institut für Mathematik, Universität Zürich, Winterthurerstr. 190, 8057 Zürich, Switzerland e-mail: joseph.ayoub@math.uzh.ch

Eingegangen 22. Februar 2013 